\title{
Comparação de modelos matemáticos para o traçado de curvas granulométricas
}

\author{
Euzebio Medrado da Silva(1), Jorge Enoch Furquim Werneck Lima ${ }^{(1)}$, \\ Lineu Neiva Rodrigues ${ }^{(1)}$ e Juscelino Antônio de Azevedo ${ }^{(1)}$
}

(1)Embrapa Cerrados, BR-020 km 18, Caixa Postal 08223, CEP 73301-970 Planaltina, DF. E-mail: euzebio@cpac.embrapa.br, jorge@cpac.embrapa.br, lineu@cpac.embrapa.br, juscelin@cpac.embrapa.br

Resumo - A distribuição granulométrica de partículas sólidas é essencial para as áreas de material de construção, mecânica dos solos, física dos solos, hidrossedimentologia, entre outras. As técnicas utilizadas na avaliação da distribuição granulométrica de amostras resultam em valores pontuais, dependendo de posterior interpolação para o traçado da curva granulométrica e a obtenção de diâmetros característicos específicos. A transformação de valores pontuais em funções contínuas pode ser realizada por meio de modelos matemáticos. Entretanto, há poucos estudos com a finalidade de determinar o melhor modelo para o ajuste de curvas granulométricas. O objetivo deste trabalho foi testar e comparar 14 diferentes modelos passíveis de utilização no traçado da curva granulométrica de partículas sólidas com base em quatro pontos medidos. O parâmetro de comparação entre os modelos foi a soma de quadrado dos erros entre os valores medidos e calculados. Os modelos mais recomendados no traçado da curva granulométrica, a partir de quatro pontos, são os de Skaggs et al. 3P, Lima \& Silva 3P, Weibull 3P e Morgan et al. 3P, todos com três parâmetros de ajuste.

Termos para indexação: textura do solo, granulometria, regressão não linear, curva de crescimento.

\section{Comparison of mathematical models for fitting particle-size distribution curves}

Abstract - Particle-size distribution is fundamental for characterizing construction materials, soil mechanics, soil physics, sediment-flux in rivers, and others. The techniques used to determine the particle-size distribution of a sample are point-wise, demanding posterior interpolation to fit the complete particle-size distribution curve and to obtain values of specific diameters. The transformation of discrete points into continuous functions can be made by mathematical models. However, there are few studies to determine the best model to fit particle-size distribution curves. The objective of this work was to test and compare 14 different models with feasibility to fit the cumulative particle-size distribution curve based on four measured points. The parameter used to compare the models was the sum of the square errors between the measured and calculated values. The most recommendable models to fit the particle-size distribution curve, based on four discrete points, are Skaggs et al. 3P, Lima \& Silva 3P, Weibull 3P, and Morgan et al. 3P, all using three fitting parameters.

Index terms: soil texture, soil fractions, non-linear regression, growth curves.

\section{Introdução}

O conhecimento sobre a distribuição granulométrica de partículas sólidas é essencial para várias aplicações. Assim, é por meio da análise granulométrica que se determina a textura dos solos, parâmetro fundamental na inferência do potencial de compactação, da disponibilidade de água, da aeração, da condutividade do solo ao ar, à água e ao calor, da infiltração e da redistribuição de água (Prevedello, 1996). Além disso, como os processos de erosão, transporte e deposição de partículas sólidas são dependentes, entre outras grandezas, da granulometria dos materiais expostos e carreados, o traçado da curva granulométrica é essencial na hidrossedimentologia, possibilitando a obtenção de determinados diâmetros característicos das amostras, fundamentais na estimativa do deslocamento de sedimentos em bacias hidrográficas (Carvalho et al., 2000).

Em geral, as técnicas utilizadas no levantamento de dados granulométricos resultam em valores pontuais, definindo a proporção com que os diferentes tamanhos de partículas sólidas ocorrem em determinada amostra. Existem diversas classificações para definir as escalas de tamanho dessas partículas, tais como as propostas pelo Departamento de Agricultura dos Estados Unidos (Estados Unidos, 1951) e pela Sociedade Brasileira de Ciência do Solo (Lemos \& Santos, 1984). A existência de diferentes escalas complica a organização dessas 
informações em uma base de dados única. Para solucionar esse problema, Nemes et al. (1999) testaram diferentes procedimentos para compatibilizar os valores pontuais da distribuição granulométrica de várias bases de dados de solos europeus.

A transformação de valores pontuais em funções contínuas tem sido objeto de vários estudos, conforme revisão de Hwang II et al. (2002), na qual se destacam as funções log-normais e as do tipo curvas de crescimento, como as freqüentemente utilizadas para representar curvas granulométricas de partículas de solo. Os modelos mais adequados à representação da curva granulométrica devem ser capazes de traçar uma função contínua em forma de "S", a exemplo dos modelos de curvas de crescimento descritos por Haverkamp \& Parlange (1986), Fredlund et al. (2000), Naime et al. (2001) e Lima \& Silva (2002).

Hwang II et al. (2002) compararam sete modelos: cinco log-normais, com um, dois e três parâmetros (Buchan et al., 1993); Gompertz, com quatro parâmetros (Nemes et al., 1999); e Fredlund, com quatro parâmetros (Fredlund et al., 2000), utilizando 1.387 amostras de solos coreanos. Esses autores concluíram que o modelo de Fredlund apresentou o melhor desempenho na maioria dos solos estudados, aumentando sua performance com o incremento no teor de argila e que o modelo de Gompertz, mesmo com quatro parâmetros, foi apenas um pouco melhor do que os demais, com dois e três parâmetros. Observaram, ainda, variações no desempenho de ajuste dos modelos em função da classe de solo analisada.

O trabalho de Hwang II et al. (2002) representa importante contribuição na comparação de modelos no traçado da curva granulométrica. No entanto, seu estudo é relativamente incompleto, pois outros modelos, potencialmente adaptáveis a essa finalidade, não foram contemplados. Além disso, a comparação direta de modelos com diferentes números de parâmetros pode favorecer àqueles que os têm em maior quantidade, tornando duvidosas suas conclusões em relação à performance dos modelos testados.

O objetivo deste trabalho foi testar e comparar 14 diferentes modelos passíveis de utilização no traçado da curva granulométrica de partículas sólidas com base em quatro pontos medidos.

\section{Material e Métodos}

Foram utilizados resultados de análises ranulométricas de 130 perfis de solos descritos no Projeto RadamBrasil
(Brasil, 1973-1986), com amostras distribuídas equitativamente nas 13 classes texturais descritas por Lemos \& Santos (1984) (Figura 1). Para cada uma das treze classes texturais, 10 tipos de solos com suas respectivas frações granulométricas - argila (partículas com diâmetro menor do que $0,002 \mathrm{~mm}$ ); silte (diâmetro de partículas entre 0,002 e $0,05 \mathrm{~mm}$ ); areia fina (partículas entre 0,05 e $0,2 \mathrm{~mm}$ ); e areia grossa (partículas entre 0,2 e $2 \mathrm{~mm}$ ) - encontram-se indicadas no triângulo textural (Figura 2).

Esses dados foram utilizados no ajuste de 14 modelos matemáticos do tipo curva de crescimento, selecionados em diversas fontes, com potencial para descrição do traçado da curva granulométrica (Tabela 1). A comparação entre os modelos foi baseada na soma de quadrado dos erros verificados entre os valores observados e ajustados. Como a base de dados utilizada era composta de apenas quatro frações granulométricas, o número máximo de parâmetros no ajuste dos modelos foi restrito a três.

A determinação dos parâmetros de ajuste dos modelos aos pontos conhecidos da curva foi efetuada por meio da rotina Solver do programa Microsoft Excel. A funçãoobjetivo do processo de otimização foi descrita de forma a minimizar o valor da soma de quadrados dos erros (SQ $Q_{\text {erro }}$ ) entre os valores observados e calculados das frações acumuladas. Tratando-se de uma otimização de modelos não-lineares, a escolha dos valores iniciais dos parâmetros a serem ajustados foi fundamental na convergência do processo de otimização do mínimo global.

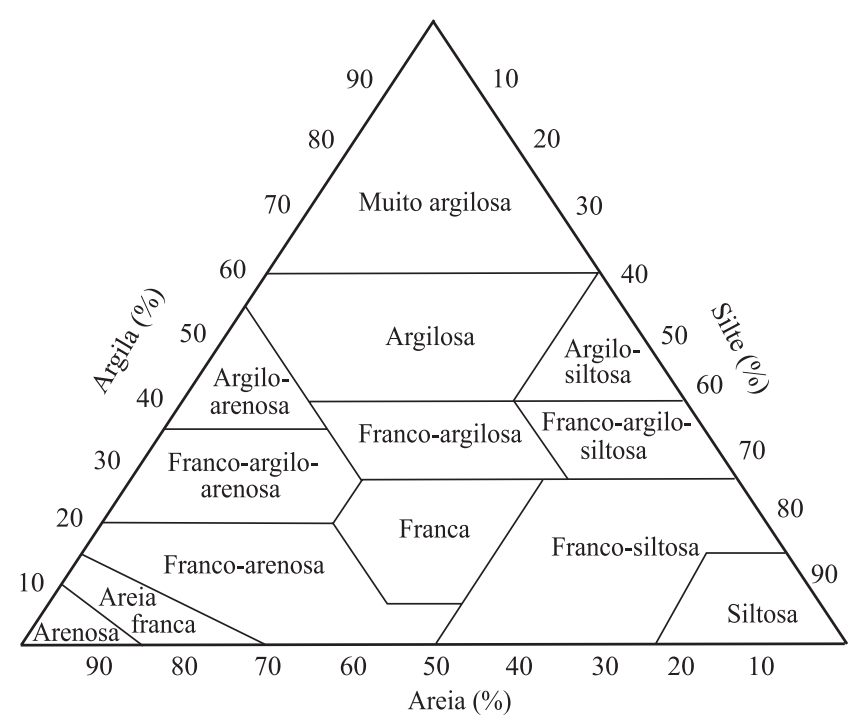

Figura 1. Triângulo de classificação textural de solos (Lemos \& Santos, 1984). 
Assim, os valores iniciais dos parâmetros na otimização foram estimados com o auxílio gráfico, observando a proximidade entre a curva gerada e os pontos medidos. Para garantir que o resultado obtido era realmente o mínimo global, o procedimento de otimização foi repetido até a estabilização do resultado da $S Q_{\text {erro }}$. Nos casos em que a curva ajustada divergia substancialmente dos pontos observados, a otimização foi refeita com outras estimativas iniciais na expectativa de melhoria do resultado.

Na comparação dos modelos, buscou-se primeiramente definir o tipo de distribuição estatística que me lhor representasse a freqüência dos 130 valores de $\mathrm{SQ}_{\text {erro }}$ resultantes da otimização de cada modelo. Para isso, foram elaborados histogramas de freqüência dos valores de $\mathrm{SQ}_{\text {erro }}$ obtidos, divididos em oito classes, segundo método descrito por Iman \& Conover (1983). Analisando a forma dos histogramas, verificou-se que a distribuição que melhor os representava foi a exponencial, fato estatisticamente comprovado por meio da medida de correlação entre os valores observados da frequiência acumulada e os calculados pelo modelo de distribuição. A significância dessa correlação foi obtida por meio do teste de significância $\mathrm{r}$ de Pearson (Levin, 1987) Esse tipo de distribuição é próprio para estabelecer o valor da variável $\mathrm{SQ}_{\mathrm{erro}}$, a partir do qual o modelo ajus-

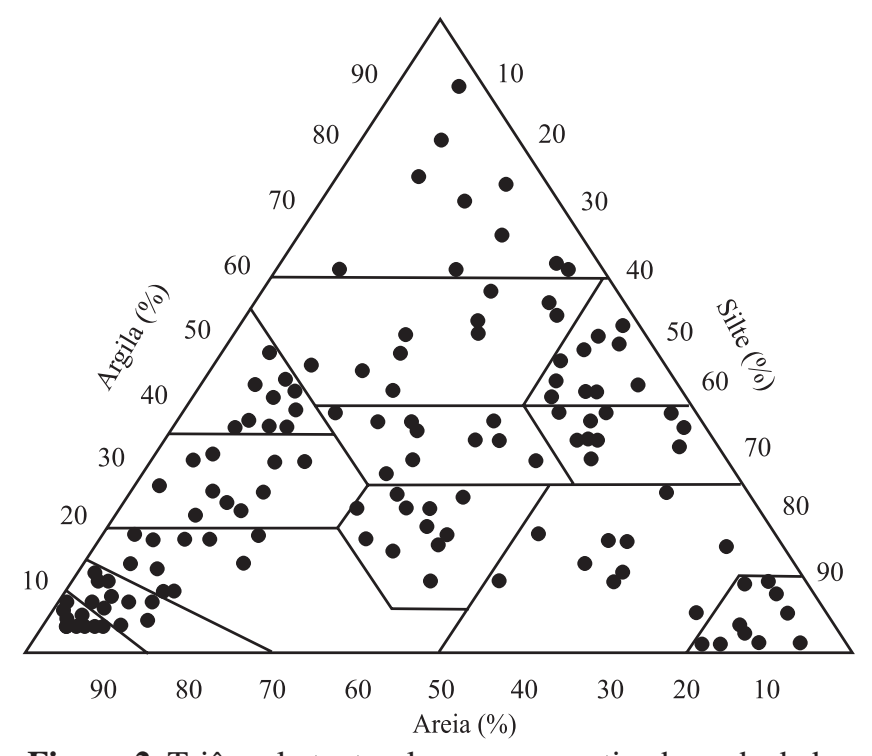

Figura 2. Triângulo textural com a respectiva base de dados utilizada neste trabalho. tado é considerado falho em determinado nível de probabilidade.

Uma vez definido o parâmetro estatístico $\lambda$ que representa o inverso da média dos $\mathrm{SQ}$ erro (Iman \& Conover, 1983), a distribuição exponencial correspondente a cada modelo ficou estabelecida. Assim, para cada modelo, foi determinado o valor de $S Q_{\text {erro }}$ correspondente ao nível crítico de $5 \%$ de probabilidade ( $S Q_{\text {erro }}$ crítico), possibilitando, dessa maneira, a comparação entre eles sob a mesma base. Por esse critério, quanto menor o SQ erro crítico, melhor o modelo. Além disso, verificou-se, para cada modelo, o número de casos em que os valores de $S Q_{\text {erro }}$ foram inferiores ao $S Q_{\text {erro }}$ crítico, ou seja, quantas vezes o modelo representou adequadamente os pontos observados com uma probabilidade de erro menor do que a do nível crítico estabelecido.

\section{Resultados e Discussão}

As frequiências acumuladas dos valores de $\mathrm{SQ}_{\text {erro }}$ obtidos no ajuste dos 14 modelos analisados encontram-se ilustradas na Figura 3. Os pontos ao longo de cada curva representam os valores calculados pela distribuição exponencial dos 130 valores de SQerro. Quanto mais a curva gerada se aproxima do eixo das ordenadas, tanto melhor o modelo. Para destacar a diferença entre os modelos, a 5\% de probabilidade, são apresentas as Figuras 3b e 3d, respectivas ampliações das Figuras 3a e 3c.

Em relação aos modelos com dois parâmetros (Figuras 3a e 3b), o Skaggs et al. (modificado) foi o que apresentou o melhor desempenho no ajuste das curvas granulométricas, resultando nos menores valores de $\mathrm{SQ}_{\text {erro }}$. Em seguida, restou um conjunto de quatro modelos (Morgan et al. 2P, Weibull 2P, Lima \& Silva 2P e Skaggs et al. 2P), que embora apresentasse desempenho inferior ao de Skaggs et al. 2P (mod), demonstrou resultados satisfatórios. Finalmente, destacaram-se os modelos Haverkamp \& Parlange 2P e Gompertz 2P, como os menos adequados para os objetivos propostos.

No que se refere aos modelos com três parâmetros (Figuras 3c e 3d), enfatizou-se a existência de um conjunto de quatro modelos com melhor desempenho (Skaggs et al. 3P, Lima \& Silva 3P, Weibull 3P e Morgan et al. 3P) e, em seguida, os modelos Richards 3P e Harverkamp \& Parlange 3P. O modelo Fredlund et al. 3P foi o que apresentou o pior resultado entre os modelos de três parâmetros. Essa classificação diverge dos resultados de Hwang II et al. (2002), no qual o modelo de 
Tabela 1. Modelos de dois (2P) e três (3P) parâmetros para o traçado da curva granulométrica nos quais $\mathrm{P}_{\mathrm{d}}$ representa o porcentual de partículas com diâmetro menor ou igual a d $(\mathrm{mm})^{(1)}$.

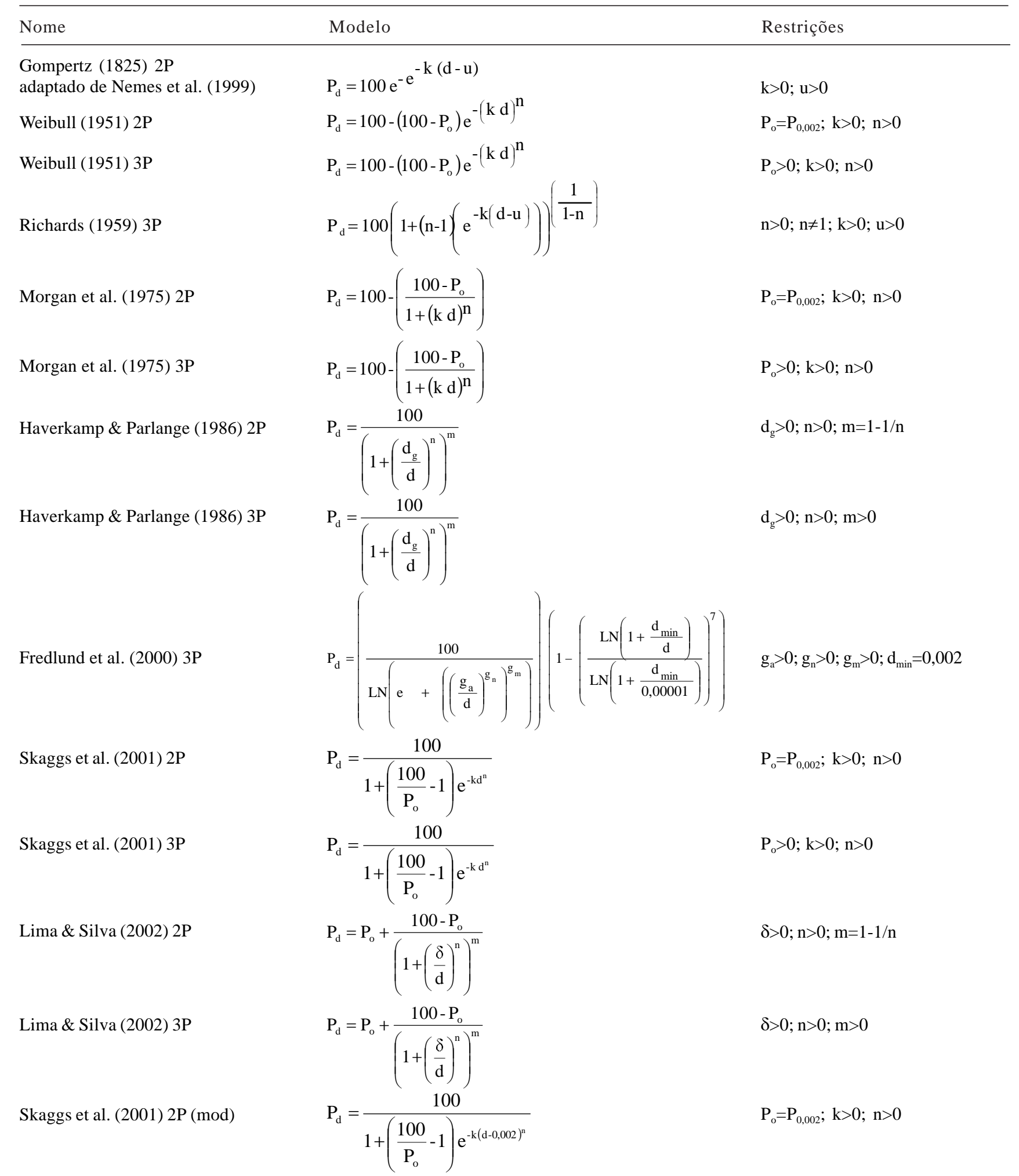

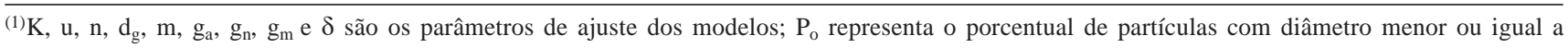
$0,002 \mathrm{~mm}$, em alguns casos fixo e, em outros, um dos parâmetros de ajuste.

Pesq. agropec. bras., Brasília, v.39, n.4, p.363-370, abr. 2004 
Fredlund et al. (2000) com quatro parâmetros foi considerado o melhor quando comparado a cinco modelos log-normais, propostos por Buchan et al. (1993) e a dois modelos (log-linear e Gompertz), usados por Nemes et al. (1999), utilizando a base de dados de solos coreanos. É provável que a escolha do conjunto de modelos testados por Hwang II et al. (2002) não tenha sido adequada, deixando de considerar modelos potencialmente melhores. Além disso, ao comparar modelos com
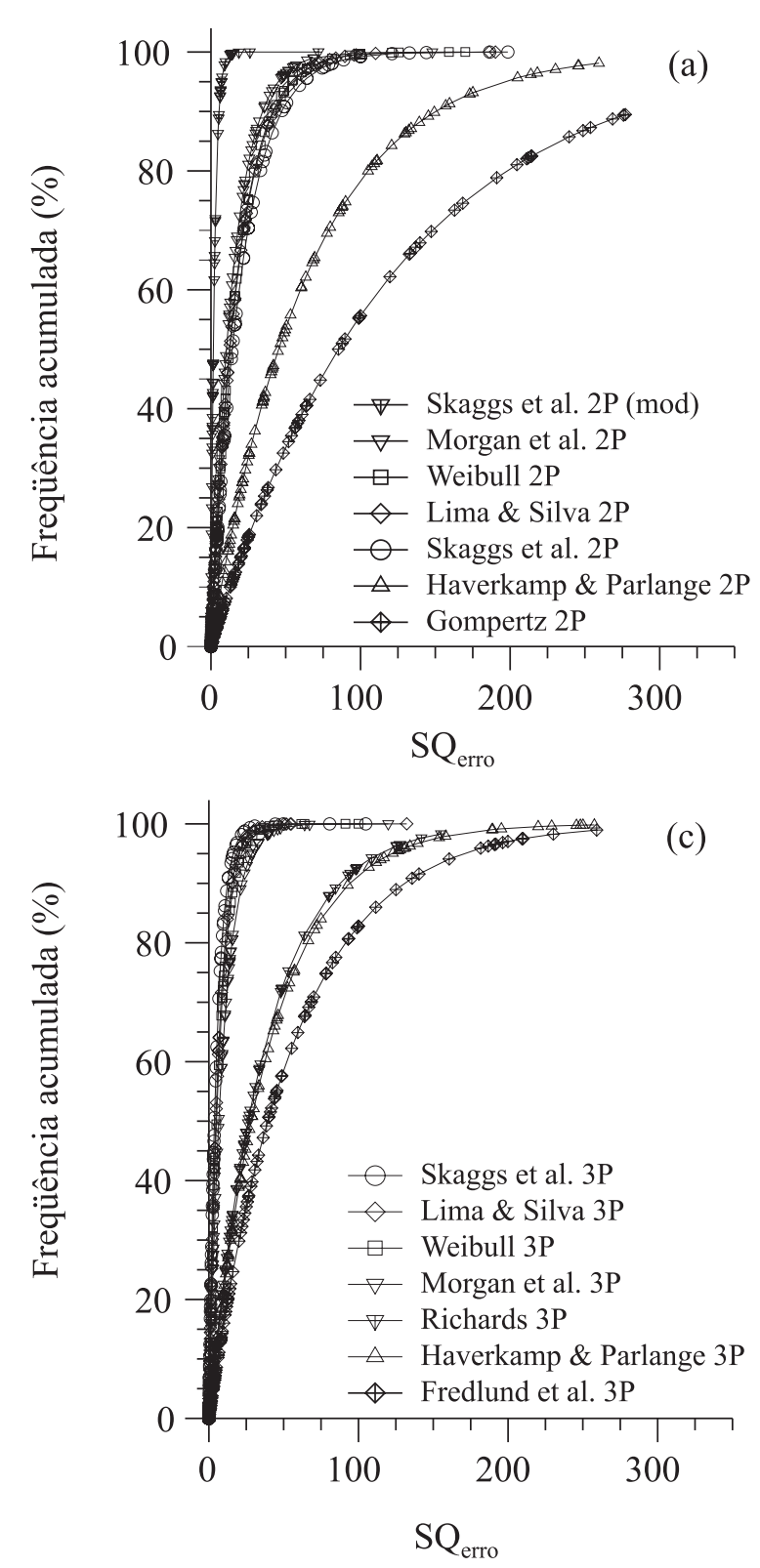

diferentes números de parâmetros de ajuste, Hwang II et al. (2002) beneficiou os modelos que os têm em maior quantidade.

Na Tabela 2, apresenta-se, em ordem crescente, a média das $\mathrm{SQ}_{\text {erro }}$ e o valor correspondente ao "SQ $\mathrm{S}$ erro crítico" obtidos de cada modelo. A "média SQerro" representa o inverso do parâmetro estatístico determinante da distribuição exponencial de cada modelo, servindo também de critério na comparação entre eles. O item
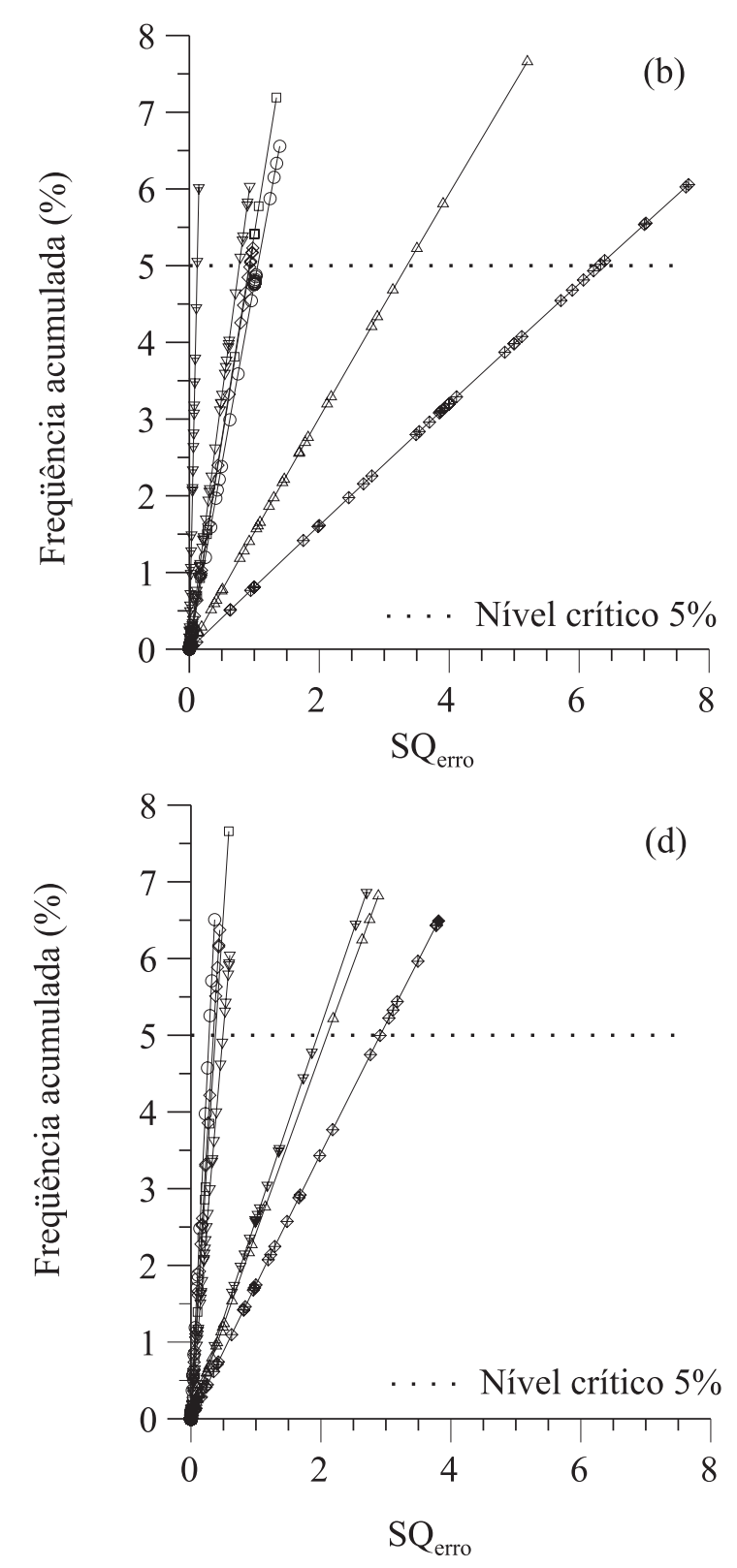

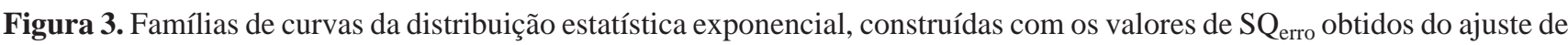
cada modelo (2P e 3P) às curvas granulométricas analisadas, ilustrando ao lado de cada caso a visão expandida da porção das curvas até o nível crítico de $5 \%$ de probabilidade. 
"casos aceitos" indica o número de vezes em que o valor de SQerro foi menor do que o "SQerro crítico". Os elevados valores de correlação obtidos e seus respectivos níveis de significância (NS\%) indicaram que os valores observados de freqüência acumulada foram explicados pela distribuição estatística exponencial.

Esperava-se que os modelos com três parâmetros (3P) apresentassem melhor desempenho do que aqueles com dois parâmetros (2P). Entretanto, como se pode observar, o modelo Skaggs et al. 2P (modificado) foi o que alcançou melhor desempenho no ajuste das curvas analisadas (Tabela 2). Além disso, alguns modelos de três parâmetros (Richards 3P, Haverkamp \& Parlange 3P e Fredlund et al. 3P) apresentaram desempenho inferior a modelos com dois parâmetros (Morgan et al. 2P, Weibull 2P, Lima \& Silva 2Pe Skaggs et al. 2P). Hwang II et al. (2002) também constataram que o maior número de parâmetros do modelo não é garantia de sucesso no ajuste de curvas granulométricas e concluíram que o modelo de Gompertz, utilizado com quatro parâmetros naquele trabalho, apresentou desempenho inferior a outros com dois e três parâmetros.

Para avaliar o traçado das curvas quanto à sua forma, selecionaram-se, para fins ilustrativos, quatro casos representativos de qualidades e anomalias encontradas no ajuste das curvas granulométricas (Figura 4). As classes texturais e composições granulométricas das amostras selecionadas foram as seguintes: arenosa $(6 \%$ argila, $4 \%$ silte, $44 \%$ areia fina, $46 \%$ areia grossa); franca (19\% argila, $41 \%$ silte, $31 \%$ areia fina, $9 \%$ areia grossa); $\operatorname{argilosa}(51 \%$ argila, $21 \%$ silte, $20 \%$ areia fina, $8 \%$ areia grossa); e argilo-siltosa (49\% argila, $42 \%$ silte, $6 \%$ areia fina, $3 \%$ areia grossa). Com vistas a melhorar a visualização das curvas, cada classe textural foi representada por dois gráficos dispostos lado a lado: o da esquerda, com os sete modelos de dois parâmetros, e o da direita, com os sete de três parâmetros.

Os modelos (2P e 3P) foram hábeis no ajuste das curvas aos pontos observados (Figura 4). Entretanto, no caso da amostra de textura arenosa, os modelos Haverkamp \& Parlange 2P e 3P, Gompertz 2P e Fredlund et al. 3P divergiram na parte inicial da curva. Na textura franca, Gompertz 2P e Richards 3P não apresentaram a flexibilidade necessária para incluir o ponto de areia fina em seu ajuste. Na textura argilosa, Gompertz 2P, Haverkamp \& Parlange 2P e Fredlund et al. 3P foram os que apresentaram maior dificuldade de ajuste. A limitação do modelo Fredlund et al. (2000), no traçado de curvas granulométricas de solos argilosos, contrariou os resultados de Hwang II et al. (2002), nos quais, com o aumento do teor de argila das amostras, o referido modelo apresentou uma performance melhorada em relação aos demais.

No caso do solo com maior teor de silte (textura argilo-

Tabela 2. Indicadores de aderência entre os valores da distribuição estatística exponencial e da freqüência acumulada observada, relativos a oito classes de $\mathrm{SQ}_{\text {erro }}$ analisadas e indicadores estatísticos correspondentes aos 130 valores individuais de $\mathrm{SQ}_{\text {erro }}$ calculados com base em cada modelo.

\begin{tabular}{|c|c|c|c|c|c|c|}
\hline \multirow[t]{2}{*}{ Modelos } & \multicolumn{3}{|c|}{ Indicadores de aderência } & \multicolumn{3}{|c|}{ Indicadores estatísticos } \\
\hline & Correlação & Teste $\mathbf{t}^{(1)}$ & $\mathrm{NS} \%{ }^{(2)}$ & Média $\mathrm{SQ}_{\text {erro }}$ & $\mathrm{SQ}_{\text {erro }}$ crítico & Casos aceitos $^{(3)}$ \\
\hline Skaggs et al. (2001) 2P (mod) & 0,97 & 10,02 & 100,00 & 2,38 & 0,122 & 84 \\
\hline Skaggs et al. (2001) 3P & 0,95 & 7,33 & 99,98 & 5,43 & 0,279 & 67 \\
\hline Lima \& Silva (2002) 3P & 0,94 & 6,83 & 99,98 & 6,73 & 0,345 & 58 \\
\hline Weibull (1951) 3P & 0,93 & 6,04 & 99,95 & 7,32 & 0,375 & 61 \\
\hline Morgan et al. (1975) 3P & 0,93 & 6,34 & 99,96 & 9,61 & 0,493 & 55 \\
\hline Morgan et al. (1975) 2P & 0,97 & 10,16 & 100,00 & 14,97 & 0,768 & 45 \\
\hline Weibull (1951) 2P & 0,93 & 6,42 & 99,97 & 17,97 & 0,922 & 36 \\
\hline Lima \& Silva (2002) 2P & 0,93 & 6,03 & 99,95 & 18,21 & 0,934 & 39 \\
\hline Skaggs et al. (2001) 2P & 0,94 & 6,89 & 99,98 & 20,53 & 1,053 & 49 \\
\hline Richards (1959) 3P & 0,92 & 5,61 & 99,93 & 38,05 & 1,952 & 41 \\
\hline Haverkamp \& Parlange (1986) 3P & 0,93 & 6,45 & 99,97 & 40,87 & 2,096 & 42 \\
\hline Fredlund et al. (2000) 3P & 0,96 & 8,68 & 99,99 & 56,81 & 2,914 & 30 \\
\hline Haverkamp \& Parlange (1986) 2P & 0,97 & 10,31 & 100,00 & 65,30 & 3,349 & 30 \\
\hline Gompertz (1825) 2P & 0,97 & 9,43 & 100,00 & 123,01 & 6,309 & 38 \\
\hline
\end{tabular}

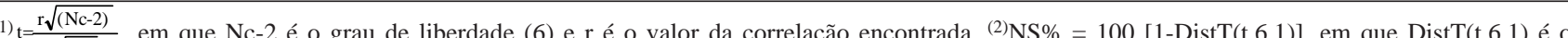
valor da distribuição unicaudal t com 6 graus de liberdade. ${ }^{(3)}$ Corresponde ao número de casos nos quais o valor da $S Q_{\text {erro }}$ é inferior ao valor crítico determinado a $5 \%$ de probabilidade. 

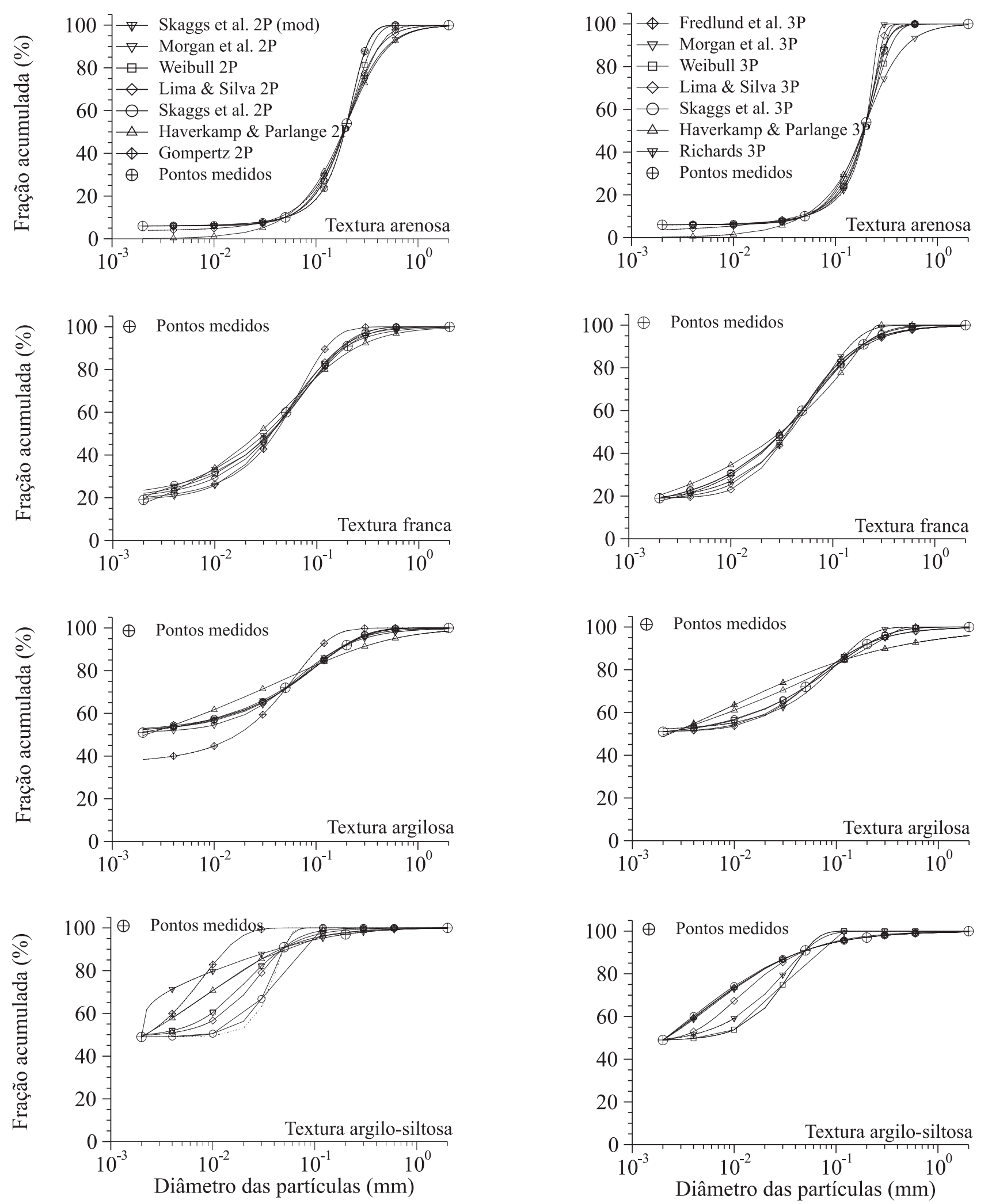

Figura 4. Conjunto de curvas geradas de quatro classes texturais com a aplicação dos diferentes modelos em uma dada amostra de solo. 
siltosa), os modelos apresentaram a maior variedade de traçados no ajuste aos pontos observados, havendo maior divergência nos modelos com dois parâmetros (Figura 4). Nesse tipo de solo, o modelo de Gompertz, apesar do formato adequado, não foi capaz de ajustar o ponto correspondente ao teor de silte. Não obstante o melhor ajuste aos pontos observados, o modelo Skaggs et al. 2P (mod) apresentou formato divergente do traçado sigmoidal esperado. Essa anormalidade também foi verificada por Skaggs et al. (2001) ao aplicar seu modelo em solos com teor de silte acima de $70 \%$, o que torna seu emprego no ajuste de curvas granulométricas com quatro pontos duvidoso.

\section{Conclusões}

1. Apesar de ter obtido a melhor classificação, o modelo Skaggs et al. 2P (modificado), em alguns casos, não reproduz o traçado sigmoidal esperado, sendo seu emprego recomendado com ressalvas.

2. Os modelos mais recomendados no traçado da curva granulométrica, a partir de quatro pontos, são Skaggs et al 3P, Lima \& Silva 3P, Weibull 3P e Morgan et al. 3P.

3. Entre os modelos de dois parâmetros, os mais recomendáveis são os de Morgan et al. 2P, Weibull 2P, Lima \& Silva $2 \mathrm{P}$ e Skaggs et al. 2P.

4. O modelo de Gompertz 2P, por seu pior desempenho, não é recomendável no traçado de curvas granulométricas a partir de quatro pontos.

\section{Agradecimentos}

Ao CNPq pela concessão da bolsa PROFIX ao terceiro autor; aos empregados da Embrapa Cerrados, que contribuíram com sugestões para a melhoria deste trabalho.

\section{Referências}

BRASIL. Ministério das Minas e Energia. Departamento Nacional de Produção Mineral. Projeto RADAMBRASIL. Rio de Janeiro, 1973-1986. (Levantamento de Recursos Naturais, v.1-34)

BUCHAN, G.D.; GREWAL, K.S.; ROBSON, A.B. Improved models of particle-size distribution: an illustration of model comparison techniques. Soil Science Society of America Journal, v.57, p.901908, 1993.
CARVALHO, N.O.; FILIZOLA JÚNIOR, N.P.; SANTOS, P.M.C.; LIMA, J.E.F.W. Guia de práticas sedimentométricas. Brasília: ANEEL, 2000. 154p

ESTADOS UNIDOS. Department of Agriculture. Soil Survey Staff. Soil survey manual. Washington, 1951. 503p. (Handbook, 18) FREDLUND, M.D.; FREDLUND, D.G.; WILSON, G.W. An equation to represent grain-size distribution. Canadian Geotechnical Journal, v.37, p.817-827, 2000.

HAVERKAMP, R.; PARLANGE, J.Y. Predicting the water-retention curve from particle-size distribution - I: sandy soils without organic matter. Soil Science Society of America Journal, v.142, p.325-339, 1986.

HWANG II, S.; LEE, K.P.; LEE, D.S.; POWERS, S.E. Models for estimating soil particle-size distributions. Soil Science Society of America Journal, v.66, p.1143-1150, 2002.

IMAN, R.L.; CONOVER, W.J. A modern approach to statistics. New York: J. Wiley, 1983. 497p.

LEMOS, R.C.; SANTOS, R.D. Manual de descrição e coleta de solo no campo. 2.ed. Campinas: Sociedade Brasileira de Ciência do Solo; Embrapa-SNLCS, 1984. 46p.

LEVIN, J. Estatística aplicada a ciências humanas. 2.ed. São Paulo: Harbra, 1987. 392p.

LIMA, J.E.F.W.; SILVA, E.M. Utilização do modelo modificado de Genuchten para o traçado da curva granulométrica. In: ENCONTRO NACIONAL DE ENGENHARIA DE SEDIMENTOS, 5., 2002, São Paulo. Anais. São Paulo: Associação Brasileira de Recursos Hídricos, 2002. p.121-125.

MORGAN, P.H.; MERCER, L.P.; FLODIN, N.W. General model for nutritional responses of higher organisms. Proceedings of the National Academy of Sciences, v.42, p.4327-4331, 1975.

NAIME, J.M.; VAZ, C.M.P.; MACEDO, A. Automated soil particle size analizer based on gamma-ray attenuation. Computer and Electronics in Agriculture, v.31, p.295-304, 2001.

NEMES, A.; WÖSTEN, J.H.M.; LILLY, A.; VOUSHAAR, J.H.O. Evaluation of different procedures to interpolate particle-size distribuitions to achieve compatibility within soil databases. Geoderma, v.90, p.187-202, 1999.

PREVEDELLO, C.L. Física do solo com problemas resolvidos. Curitiba: Salesward-discovery, 1996. 446p.

RICHARDS, F.J. A flexible growth function for empirical use. Journal of Experimental Botany, v.10, p.290-300, 1959.

SKAGGS, T.H.; ARYA, L.M.; SHOUSE, P.J.; MOHANTY, B.P. Estimating particle-size distribution from limited soil texture data. Soil Science Society of America Journal, v.65, p.1038-1044, 2001

WEIBULL, W. A statistical distribution function of wide applicability. Journal of Applied Mechanics, v.73, p.293-297, 1951 